\title{
Signal Analysis of CMP Process based on $A E$ Monitoring System
}

\author{
Sun-Joon Park', Hyun-Seop Lee', and Haedo Jeong, \\ 1 School of Mechanical Engineering, Pusan National University, 2, Busandaehak-ro 63beon-gil, Geumjeong-gu, Busan, South Korea, 609-836 \\ 2 Department of Mechanical Engineering. Tongmyong University, 428, Sinseon-ro, Nam-gu, Busan, South Korea, 608-711 \\ \# Corresponding Author / E-mail: hdjeong@pusan.ac.kr, TEL: +82-51-510-2463, FAX: +82-51-510-8442
}

KEYWORDS: Chemical mechanical planarization(CMP), Acoustic emission(AE) sensor, Fast fourier transform(FFT)

\begin{abstract}
As chemical mechanical planarization (CMP) enables local and global planarization over a wafer surface by the combined effects of chemical and mechanical interactions, process monitoring is becoming an increasingly important in-situ methodology for process control. According to the materials and process, signal characteristics were distinguishable between material and process. In this study, an acoustic emission (AE) sensor was used to measure the abrasive and molecular-scale phenomena during CMP. An AE signal was acquired using rotational equipment and adapted to two types of equipment. First, a wireless AE system consisting of wireless modules using Bluetooth was used. This system was suitable for acquiring signals in rotational equipment. However, a wireless AE system could be acquired with only Root Mean Square(RMS) signals. Second, mercury slip-ring (wired) AE systems that were suitable for rotational equipment and the acquisition of raw signals were used. The acquired raw signals could be analyzed by a Fast Fourier Transform (FFT) for abrasive and molecular-level phenomena in the CMP process. The AE signal parameters including the AE RMS, frequency, and amplitude were analyzed for abrasive and molecular-level phenomena in the CMP process. The authors analyzed the AE signals for changes in the materials and CMP process.
\end{abstract}

\section{Introduction}

Chemical mechanical planarization (CMP) enables local and global planarization over a wafer surface by the combined effects of chemical and mechanical interactions. Recently, process monitoring in CMP has become an increasingly important for process control. ${ }^{1}$ Furthermore, many defect issues are present in Cu CMP: scratch, stick-slip, etc. In the CMP process, nanosized particles abrade a wafer surface with the aid of atomic-level chemical interactions by contact with the micrometer-scale asperities of the pad, which is expressed by a wafer scale or mechanical signals that can be measured by appropriate sensing technology. In addition, the process produces different signals depending on the types of slurry, wafer, or pad and process conditions. These signals are related to the planarization results, nonuniform removal, the under-polishing of metal lines, the erosion of the dielectric material, and scratch issues. If the appropriate sensor system is chosen, the CMP process consistency can be monitored.

Acoustic emission (AE) monitoring ${ }^{2}$ is one of the most effective methods for process monitoring. All of the existing AE monitoring methods can be divided into signal-based approaches, where the measured signal values are compared to predefined signal values; model-based approaches, where process models are determined either empirically or from physical relationships; and classification-based approaches, where a feature vector is determined from a certain class of quality features. ${ }^{3}$

The important function of the monitoring system is to monitor the process consistency. Process inconsistency in CMP originates from several sources such as the variation in the material properties of the incoming pad, the aging of slurry, changes in the pad profile, and the slurry filter life. Such variations directly affect CMP performance, leading to inconsistencies in the planarization results, nonuniform removal, the under-polishing of metal lines, the erosion of dielectric the material, and scratch issues. Thus, an important function of the measurement system should be monitoring of the process consistency. ${ }^{4}$

In this paper, an $\mathrm{AE}$ sensor was implemented in $\mathrm{CMP}$ equipment. $\mathrm{AE}$ sensors have been widely used in industry ${ }^{5,6}$ for the acquisition of high-frequency signals. An acoustic spectrum may have numerous frequencies corresponding to the elastic and plastic deformation of subsurface material layers, microscratching, microfatigue, microcorrosion, and delamination, which occur during polishing. ${ }^{7}$ 
Therefore, in this study, we propose two methods for AE monitoring systems to obtain an AE signal from the CMP process.

First, we applied a wireless AE system obtained from a rotating machine, as in CMP. A wireless AE system has the advantage of being able to obtain signals from the rotating machine easily. However, this signal is transmitted; thus, there is a limit to the amount of data that is transmitted owing to the change in the RMS value. This system is suitable for wireless $\mathrm{AE}$ after inferring the change in the process conditions due to the RMS signal change; however, this system is not suitable for the detection of fine signals generated in the wafer surface.

Second, we applied a wired AE system using a mercury slip ring for $\mathrm{AE}$ monitoring during the CMP process. This system has the disadvantage of being limited in speed in comparison to a structurally fixed wireless AE system. However, this system has the advantage of being able to detect a particular frequency range for the raw AE signal through an FFT analysis. This study carried out a raw AE signal analysis and an FFT analysis of the acquired signal using a wired AE system.

\section{Signal Sources}

In the CMP process, nanosized particles abrade a wafer surface with the aid of atomic-level chemical interactions via contact with the micrometer-scale asperities of the pad, which is pressed down by a wafer-scale force. These interactions produce physical or chemical signals that can be measured by the appropriate sensing technology. In addition, the process produces different signals depending on the types of slurry, wafer, or pad and process conditions. ${ }^{9}$ Therefore, the process consistency can be monitored if the appropriate sensor system is chosen. As the scale becomes smaller, higher frequency signals are induced because the number of contacts with asperities or the number of abrasive particles increases accordingly, as shown in Fig. 1. Therefore, a sensor with a higher bandwidth is preferable to study the fundamental process physics generated from microscale phenomena. ${ }^{4}$ In this study, we obtain the $\mathrm{AE}$ sensor signal and investigate the effects of the CMP process by using the acquired signal through a signal analysis.

\section{Experiment}

\subsection{Rotational AE Monitoring System}

In this study, an $\mathrm{AE}$ monitoring system is used to detect an $\mathrm{AE}$ signal during the polishing process. As depicted in Fig. 2, the $\mathrm{AE}$ sensor is directly attached to the backside of the wafer. If the $\mathrm{AE}$ sensor is not in contact with the wafer, it is difficult to acquire a highfrequency level signal during the CMP process. However, the CMP process consists of two rotating parts: the head and plate. In these systems, the cable of the AE sensor can be twisted; thus, we suggest two types of equipment. The first type uses a Bluetooth module attached to the polishing head. Then, the acquired AE signal was transferred to a Bluetooth receiver. These Bluetooth modules consisted of an amplifier, a filter, an RMS-DC converter, and a microcontroller. The second type uses a mercury slip-ring system attached to the upper part of the head. Then, the AE signal acquired during the CMP process was transferred to a digital board through the mercury slip ring

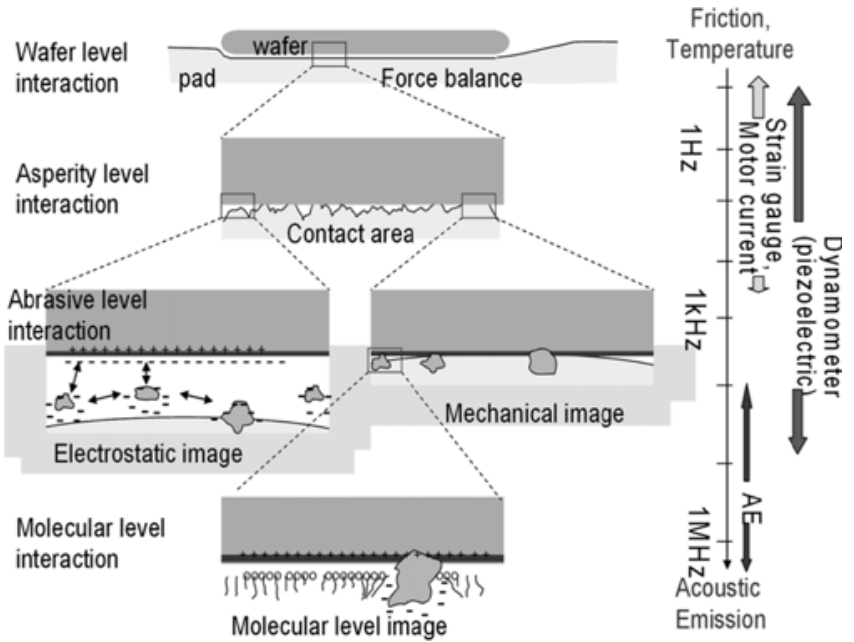

Fig. 1 Signal sources at various scales ${ }^{4}$

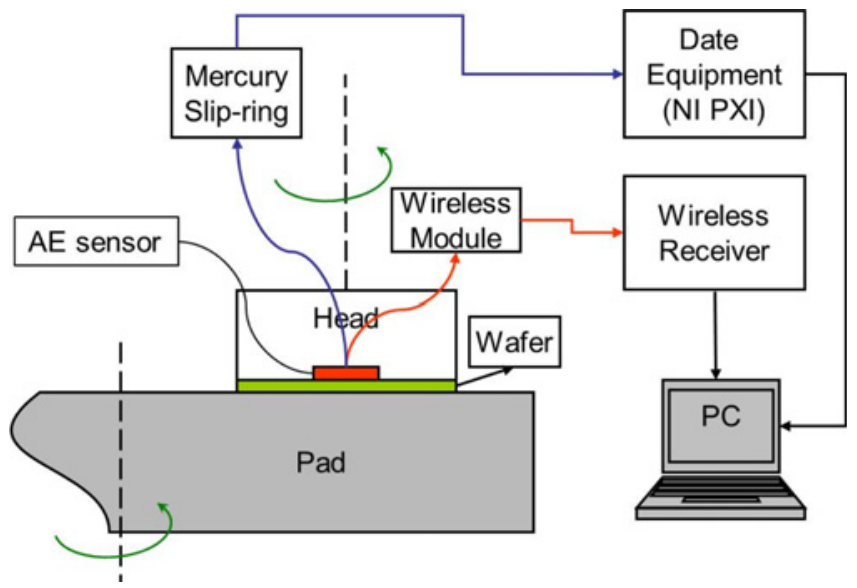

Fig. 2 Schematic of the wireless and wired AE monitoring system

(Asiantool, A2S), known to have a low-contact-resistance characteristic within $1 \mathrm{~m} \Omega$ and $\mathrm{A} 2 \mathrm{~S}$ can be applied at rotational speeds to up to 200 rpm. During the CMP process, the AE signal was measured with a widebandwidth $\mathrm{AE}$ sensor (Rectuson, M65) having a resonance-frequency range of $500 \mathrm{kHz} \pm 20 \%$, a sensitivity of $57 \pm 3 \mathrm{~dB}$, and a differentialtype output. The output $\mathrm{AE}$ signal from the $\mathrm{AE}$ sensor affixed to the backside of the wafer was amplified by a digital board (NI Co, PXI 6163) and was acquired at a sampling frequency of $1 \mathrm{MHz}$.

\subsection{Experimental Methods}

\subsubsection{Wireless AE Monitoring System}

In this study, $\mathrm{AE}$ signals are acquired using a wireless $\mathrm{AE}$ monitoring system by the AE RMS values. The signal variation is evaluated with an oxide blanket wafer. The AE RMS signal was measured during the CMP process via Bluetooth. For CMP operation, Poli-400 (GNP Technology) was used with a specially designed wafer head for sensor integration. Experiments were conducted with an IC1400 pad with ex-situ and in-situ pad conditioning. The pressure was maintained at $300 \mathrm{~g} / \mathrm{cm}^{2}$, and a constant pad rotation speed of $60 \mathrm{rpm}$ was used in all experiments.

\subsubsection{Wired AE Monitoring System}

In order to detect phenomena at the wafer surface using a high- 
Table 1 Experimental conditions

\begin{tabular}{cc}
\hline Parameter & Conditions \\
\hline Pressure & $300 \mathrm{~g} / \mathrm{cm}^{2}$ \\
\hline Velocity & $60 / 60 \mathrm{rpm}$ \\
\hline Conditioning & Ex-situ \\
\hline Pad & IC1400 \\
\hline Osillation & None \\
\hline Slurry & ILD1300 \& EPL \\
\hline Wafer & Thermal oxide \& Copper \\
\hline
\end{tabular}

frequency signal, the raw $\mathrm{AE}$ signal was monitored during the $\mathrm{CMP}$ process using a mercury slip ring. In this study, an oxide and $\mathrm{Cu}$ blanket wafer was used to detect AE signals. For CMP operation, Poli400 (GNP Technology) was used with a specially designed head for sensor integration. The AE sensor was connected by a mercury slip ring, and the process signal was acquired by a PXI-6163 (NI). The experimental conditions are listed in Table 1. All experiments were conducted with an IC1400 pad with ex-situ pad conditioning. The pressure was maintained at $300 \mathrm{~g} / \mathrm{cm}^{2}$, and constant head/platen rotation speeds of 60/60 rpm were used in all experiments. Two types of slurry were used: ILD 1300 (oxide CMP) and EPL 3-wt\% $\mathrm{H}_{2} \mathrm{O}_{2}(\mathrm{Cu}$ CMP). The slurry and DI water were supplied at a constant rate of $30 \mathrm{~mL} / \mathrm{min}$. Diamond abrasives were used as an abrasive with a particle size of $30-40 \mu \mathrm{m}$.

\section{Results and Discussion}

\subsection{Wireless AE Monitoring}

The AE RMS variation during CMP with polishing and conditioning is plotted in Fig. 3. Fig. 3 clearly shows the change in the polishing and in-situ process. In addition, Fig. 4 shows the AE RMS variation according to the change in velocity $(100,150,200,250$, and $300 \mathrm{rpm})$. These results show the variation in the process change. The AE RMS signal was measured to detect a transition signal from the oxide CMP process. A sharp transition in the AE RMS signal was observed during the change in process conditions. The transition is believed to be directly related to the variation in the process with velocity, ex-situ, and in-situ, etc. The AE RMS signal during polishing caused an increase in the energy required to initiate material removal. However, these results are difficult to explain the phenomena at the wafer surface. Because the AE RMS value carries information regarding the raw $\mathrm{AE}$ signal power during each interval of time, a reduction in the dynamic range leads to a loss of possibly valuable information. Therefore, we required a new method to analyze the raw $\mathrm{AE}$ signal in the high-frequency range.

\subsection{Wired AE Monitoring}

\subsubsection{Raw AE Signal Analysis}

$\mathrm{CMP}$ is applied to a wired $\mathrm{AE}$ monitoring system using a slip ring to obtain the raw AE signal. Fig. 5 shows the raw AE signals when using a slurry, DIW, and diamond particles in the $\mathrm{Cu}$ and oxide CMP process. $\mathrm{Cu}$ CMP is easily classified depending on each abrasive's (DIW, diamond abrasive, slurry) raw AE signals. The polishing results could also be confirmed with the naked eye, and many scratches were present on the surface of the $\mathrm{Cu}$ wafer. However, oxide CMP was

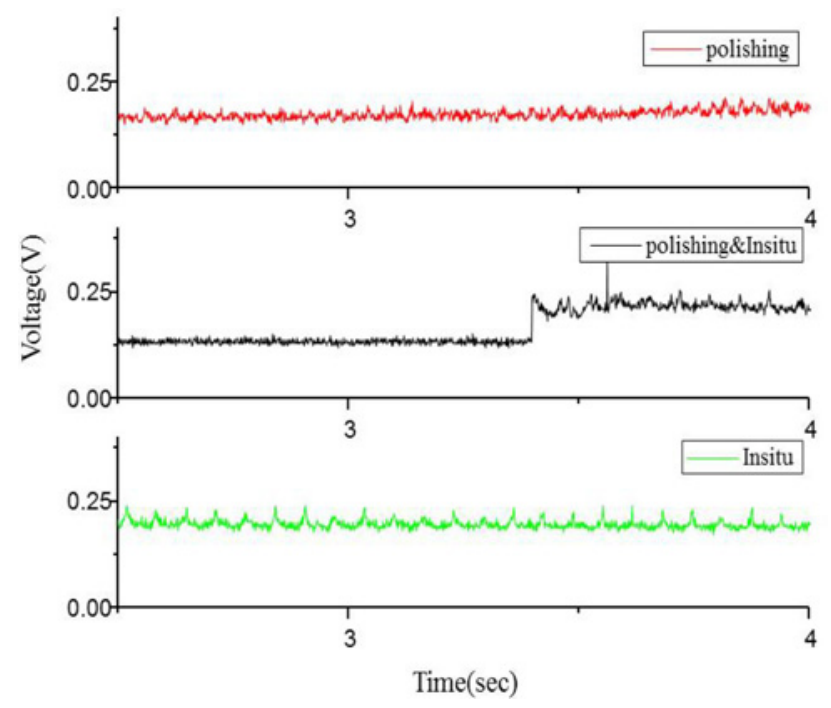

Fig. 3 Variation in the AE RMS signal during oxide CMP for ex-situ and in-situ processes

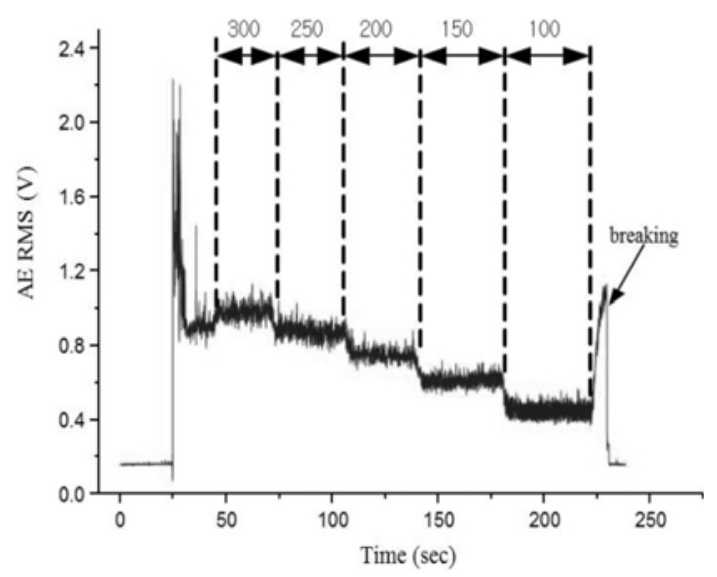

Fig. 4 Variation in the AE RMS signal for a change in head speed

confirmed to cause no substantial changes in the AE signal source. The polishing results rarely exhibited visually identifiable scratches in the surface of the oxide wafer. It can be seen that the defects caused by particles are generated relatively easily in the soft $\mathrm{Cu}$ wafer. Compared with the oxide wafer, it was confirmed that wired $\mathrm{AE}$ monitoring system can be detected as the raw signal of such defects. These results can be estimated by the correlation between a defect and the raw $\mathrm{AE}$ signals occurring during the CMP process. In particular, further research is necessary on the relationship between defects and particles because the $\mathrm{Cu} C \mathrm{CP}$ wafer characteristics change the original $\mathrm{AE}$ signal according to different abrasive particles. However, it is difficult to determine the frequency characteristic of the $\mathrm{AE}$ signals from an analysis of the raw AE signals alone. To investigate the relationship between the polishing results, an additional frequency analysis using the FFT was performed for each signal to determine the signals that occur at a particular frequency.

\subsubsection{FFT Analysis of the AE Signal}

The raw AE signals are shown in Fig. 5 for various CMP processes according to time. However, the acquired raw AE signal is needed for analysis by FFT to find the frequency characteristics of the wafer 


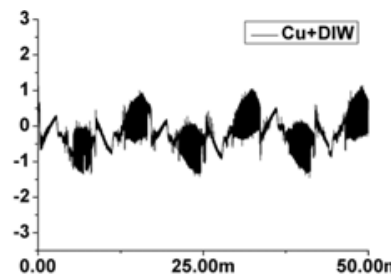

(a)

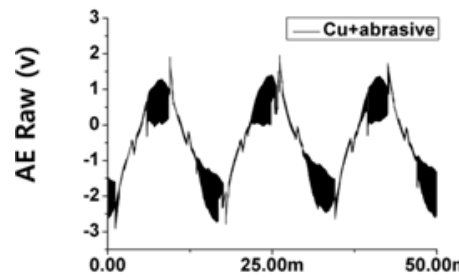

(b)

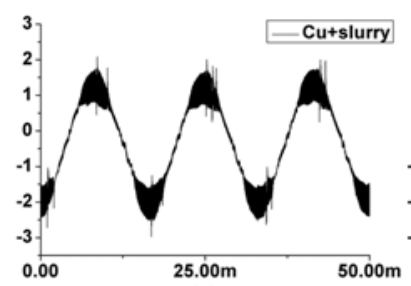

(c)

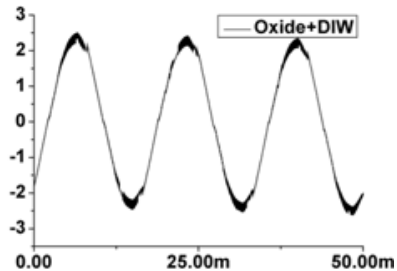

(d)

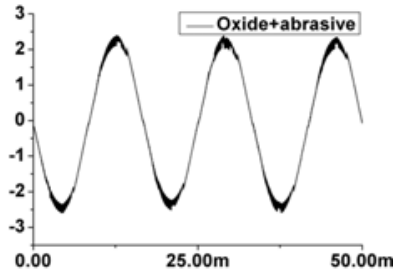

(e)

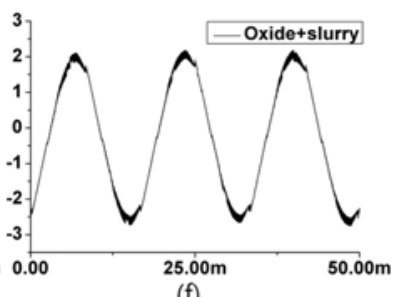

(f)
Time (s)

Fig. 5 Acquired raw AE signals in $\mathrm{Cu}$ and oxide CMP

surface during CMP. Therefore, an FFT analysis was performed with raw AE signals. Fig. 6 shows the results of the FFT analysis for $\mathrm{Cu}$ and oxide CMP. In this study, we used the Hamming window function in (1) as a function from the FFT window function. This has the advantage of a precise amplitude but the disadvantage of possible errors. By reducing the nonperiodicity in the signal period by reducing the frequency leakage in the FFT frequency analysis, the Hamming window function has a wider spectral region. This improves the resolution of the frequency. In (1), $N$ is the number of the section data. In the equiripple sense, the optimal values for the coefficients are $\alpha=0.53836$ and $\beta=0.46164 .{ }^{8}$ The FFT analysis is performed at a 1MHz sampling frequency.

$$
w[n]=\alpha+\beta \times \cos \left(\frac{2 \pi n}{N-1}\right)
$$

Fig. 6(a) shows the FFT analysis results with diamond particles (30$40 \mu \mathrm{m}$ ) in the $\mathrm{Cu}$ and Oxide CMP process. $\mathrm{Cu}$ CMP is larger than the amplitude variation of the frequency oxide CMP. The appearance and change in the frequency around the area as a whole, in particular, in less than $120 \mathrm{kHz}$ was $\mathrm{Cu} \mathrm{CMP}$ in a change in the amplitude significantly different others be found. In addition, significant changes in amplitude are confirmed to appear in the vicinity of 20 and $40 \mathrm{kHz}$; however, the frequency characteristics are not founded in the oxide CMP.

Fig. 6(b) shows the FFT analysis results of the signal when adding the slurry to the $\mathrm{Cu}$ and oxide CMP processes. The analysis was confirmed to appear in the vicinity of a large change in the amplitudes of 60,80 , and $100 \mathrm{kHz}$. However, because different types of slurry are used to polish the two different wafers, it is necessary to compare with the polishing results. In other words, additional studies are needed on the relationship between mechanical and chemical polishing.

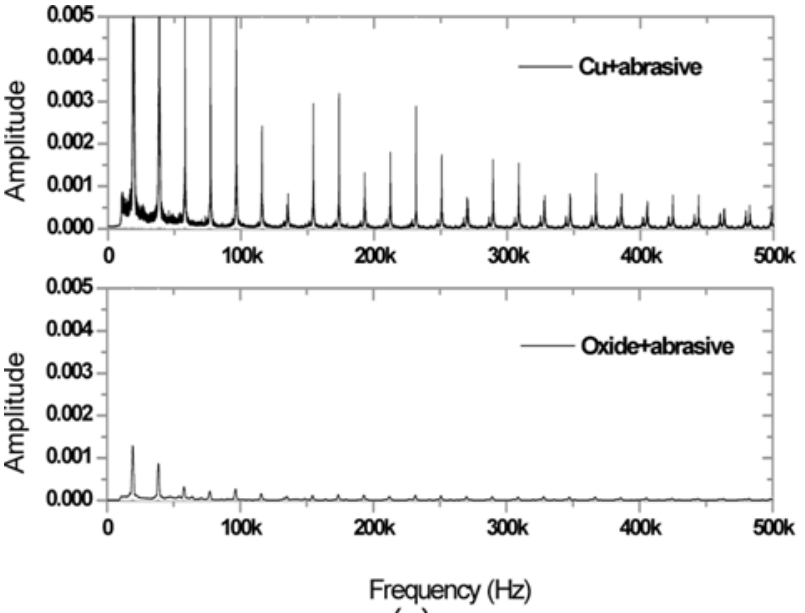

(a)
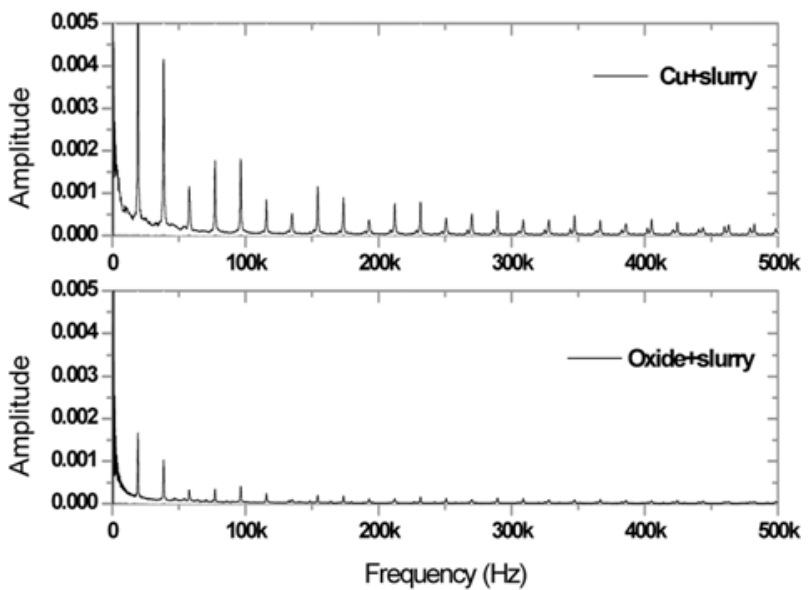

(b)

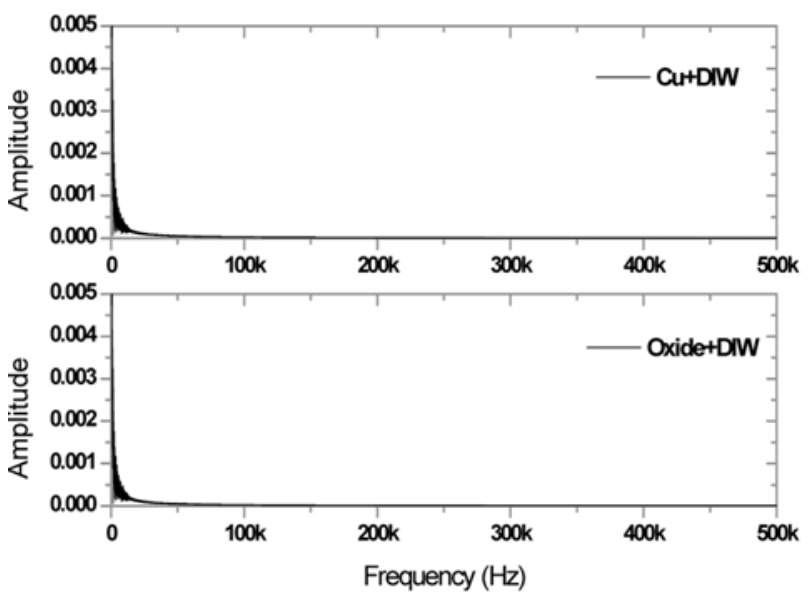

(c)

Fig. 6 FFT results: (a) with slurry (EPL $3 w t \%$ and ILD1300), (b) with abrasive (diamond abrasive), and (c) with DI water

Fig. 6(c) shows the FFT analysis results of the signal when adding DIW to the $\mathrm{Cu}$ and Oxide CMP process. However, the frequency analysis results were not found for a particular frequency band signal. It can be confirmed that the lack of signal detection is simply due to the friction between the wafer and the pad. It is clearly shown that the signals generated by the abrasive particles can be involved in the detection signals but the signals generated by CMP equipment can not be involved in detection signals. On the basis of these results, it was confirmed that the occurrence frequency of the analysis of the signal is 
different depending on the type of fine abrasive particles in the CMP process and the amount of injection. Further, a distinguished signal is detected by using the $\mathrm{AE}$ monitoring system.

\section{Conclusions}

In this study, we apply an $\mathrm{AE}$ sensor to monitor the CMP process and propose two $\mathrm{AE}$ monitoring systems for signal acquisition: wireless and wired $\mathrm{AE}$ systems. The wireless $\mathrm{AE}$ monitoring system rotates with the head of CMP equipment, so, it has no effect on the change of CMP process. Therefore, it is determined to be useful for CMP process change detection. However, detection of CMP process change can be detected with enough monitoring of friction or temperature. The wireless $\mathrm{AE}$ monitoring can be subject to transmission are converted to RMS values of the wireless transmission characteristic. However, an obtained signal is difficult to find the fine frequency that occurs in the wafer surface because it is not possible to the FFT analysis. Thus, we applied a wired AE monitoring system using a slip ring. Problems occur that are restricted to the rotation speed of the slip ring used in the AE wired system. For such problems, it is possible to obtain the raw AE signal by using the wired $\mathrm{AE}$ monitoring system. In addition, it was confirmed that the signal characteristics vary for different types of slurry when using oxide and $\mathrm{Cu}$ wafers. Further, two types of signals are used to analyze the fine signal generated in the wafer surface. It was confirmed that it is possible to detect the fine signal generated from the surface rather than the influence of the CMP process via a wired AE signal. In addition, the polishing characteristics could be inferred through the raw AE signal analysis and FFT analysis. Additional research is necessary to further consider the variations in the wafer and consumables.

\section{REFERENCES}

1. Berman, M., Bibby, T., and Smith, A., "Review of in Situ \& In-Line Detection for CMP Applications," Semiconductor Fabtech, $8^{\text {th }}$ Ed., pp. 267-274, 1998.

2. Lee, Y., Chang, A. K., and Dornfeld, D. A., "Acoustic Emission Monitoring for the Diamond Machining of Oxygen-Free HighConductivity Copper," Journal of Materials Processing Technology, Vol. 127, No. 2, pp. 199-205, 2002.

3. Wang, L. and Gao, R. X., "Condition Monitoring and Control for Intelligent Manufacturing,” Springer, 2006.

4. Jeong, H., Kim, H., Lee, S., and Dornfeld, D., "Multi-Sensor Monitoring System in Chemical Mechanical Planarization (CMP) for Correlations with Process Issues," CIRP Annals-Manufacturing Technology, Vol. 55, No. 1, pp. 325-328, 2006.

5. Karpuschewski, B., Wehmeier, M., and Inasaki, I., "Grinding Monitoring System based on Power and Acoustic Emission Sensors," CIRP Annals-Manufacturing Technology, Vol. 49, No. 1, pp. 235-240, 2000.
6. Iturrospe, A., Dornfeld, D., Atxa, V., and Manuel, A. J., "Bicepstrum Based Blind Identification of the Acoustic Emission (AE) Signal in Precision Turning," Mechanical Systems and Signal Processing, Vol. 19, No. 3, pp. 447-466, 2005.

7. Choi, J., Lee, D., Kim, H., and Dornfeld, D., "In-Situ Acoustic Emission Monitoring of Surface Chemical Reactions for Copper CMP,” Proc. of the CMP-MIC Conference, pp. 415-422, 2005.

8. Enochson, L. D. and Otnes, R. K., "Programming and Analysis for Digital Time Series Data," Shock and Vibration Information Center, p. $277,1969$.

9. Lee, H., Dornfeld, D., and Jeong, H., "Mathematical Model-Based Evaluation Methodology for Environmental Burden of Chemical Mechanical Planarization Process," Int. J. Precis. Eng. Manuf.Green Tech., Vol. 1, No. 1, pp. 11-15, 2014. 